\title{
IN-PLANE CONDUCTIVITY ANISOTROPY IN UNDERDOPED CUPRATES IN THE SPIN-CHARGE GAUGE APPROACH
}

\author{
P.A. Marchetti \\ Dipartimento di Fisica "G. Galilei", INFN, I-35131 Padova, Italy \\ G. Orso \\ International School for Advanced Studies (SISSA), Via Beirut, 34014 Trieste, Italy \\ Z.B. $\mathrm{Su}$ and $\mathrm{L} . \mathrm{Yu}$ \\ Institute of Theoretical Physics and Interdisciplinary Center of Theoretical Studies, \\ Chinese Academy of Sciences, 100080 Beijing, China
}

(Dated: November 21, 2018)

\begin{abstract}
Applying the recently developed spin-charge gauge theory for the pseudogap phase in cuprates, we propose a self-consistent explanation of several peculiar features of the far-infrared in-plane AC conductivity, including a broad peak as a function of frequency and significant anisotropy at low temperatures, along with a similar temperature-dependent in-plane anisotropy of DC conductivity in lightly doped cuprates. The anisotropy of the metal-insulator crossover scale is considered to be responsible for these phenomena. The obtained results are in good agreement with experiments. An explicit proposal is made to further check the theory.

PACS numbers: $71.10 . H f, 71.27 .+\mathrm{a}, 74.25 . \mathrm{Fy}, 74.25 . \mathrm{Gz}$
\end{abstract}

The normal state properties of cuprate superconductors have shown a number of unexpected features, particularly in underdoped regime, where the pseudogap effects are pronounced $\stackrel{1}{\underline{m}}$ One of the most recent surprises is the strong in-plane temperature-dependent anisotropy of DC conductivity, observed in lightly doped untwinned LSCO and YBCO crystals $\stackrel{2}{2}$ The maximal anisotropy $\rho_{b} / \rho_{a}-1$ reaches $50 \%$ for $3 \%$ doped LSCO which is far too big compared with the orthorhombicity (up to $1.7 \%$ ) $\stackrel{2}{=}$ A "natural" explanation of this unusual behavior would be the "self-organized" charge stripe structure, proposed by a number of authors 3.4 which was also suggested to be responsible for the occurrence of superconductivity ${ }^{5}$ The conductivity is indeed higher in the stripe direction (along the $a$-axis) $\underline{\underline{6}}$ Meanwhile, Dumm et al $l^{\underline{7}}$ recently measured the in-plane AC conductivity of untwinned $3 \%$ and $4 \%$ doped LSCO crystals. In the far infrared region at high temperatures, the data are consistent with a simple Drude model. Below $80 \mathrm{~K}$, a broad peak appears at finite frequencies $\left(\Omega \sim 100 \mathrm{~cm}^{-1}\right)$ bearing a close resemblance to the peak found in temperature-dependent DC conductivity for the same composition; a significant $a$ $b$ resistivity anisotropy is observed in complete analogy with the DC case $\stackrel{2}{2}^{2}$ These AC data seem also to support the presence of charge stripes.

However, there are several substantial difficulties in the intuitive "rivers of charge" interpretation: The meanfield theory predicts the statically charge-ordered state is an insulator,,$\frac{3}{3}$ while experimentally these lightly doped cuprates show metallic behavior at high temperatures. Also, the observed anisotropy ratio is too small compared with quasi-one-dimensional (Q1D) conductors, usually showing order-of-magnitude bigger conductivity in the chain direction. To avoid these difficulties, the "electronic liquid crystal" scenario of meandering stripes 5.8 is invoked to induce metallic conductivity and to reduce the expected anisotropy. This does not solve the problem, either. In fact, a closer examination of the data 2.7 reveals that the anisotropy effect is most pronounced in the limit $\omega, T \rightarrow 0$, in contradiction with the fluctuating stripe picture: One would anticipate much bigger effect of anisotropy at some characteristic frequencies of the stripe fluctuations, rather than the static limit. This was also pointed out in Ref. 7. Also, as evident from the experimental curves $\stackrel{2.7}{=}$ the major source of the inplane conductivity anisotropy is due to the shift of the Metal-Insulator Crossover (MIC) scale, and a stronger anisotropy is found in the localized, instead of metallic regime. Up to now, many authors attribute this MIC to the disorder effect, $\stackrel{9}{\underline{9}}$ or more specifically to localization in Q1D systems ${ }^{7}$ However, there is a fundamental difficulty in that approach: There is only one mobility edge in disorder-induced localization for anisotropic systems $\stackrel{10}{ }$, at least within the scaling theory. This means the system cannot be localized in one direction, while delocalized in another.

Recently, we have developed a spin-charge gauge approach to describe the pseudogap phase in cuprate superconductors, particularly focusing on the MIC phenomena $\stackrel{11.12}{1 n}$ this approach ${ }^{11}$ based on spin-charge decomposition applied to the $2 \mathrm{D} t-J$ model, the spinon dynamics is described by a non-linear $\sigma$-model with a theoretically derived mass gap $m_{s} \sim J(\delta|\ln \delta|)^{1 / 2}$, where $J$ is the exchange integral, $\delta$ the doping concentration; the holon is fermionic with "small" Fermi surfaces $\left(\epsilon_{F} \sim t \delta\right)$ (with $t$ as the hopping integral) centered around $( \pm \pi / 2, \pm \pi / 2)$ in the Brillouin zone and a "Fermiarc" behavior for the spectral weight. Both holons and spinons are strongly scattered by gauge fluctuations. As an effect of gauge interaction, the spinon mass picks up 
a dissipative term: $m_{s} \rightarrow M_{T}=\left(m_{s}^{2}-i c T / \chi\right)^{1 / 2}$, where $\chi \sim t \delta^{-1}$ is the diamagnetic susceptibility and $c$ a numerical constant. This shift in turn introduces a dissipation in the spinon-gauge sector, whose behavior dominates the low energy physics of the system. The competition between the mass gap and the dissipation is responsible for the MIC, giving rise to a broad peak in the DC conductivity. At low temperatures the antiferromagnetic (AF) correlation length $\xi \sim m_{s}^{-1}$ is the determining scale of the problem, leading to localizing behavior, while at higher temperatures, the de Broglie wave length $\lambda_{T} \sim(\chi / T)^{1 / 2}$ becomes comparable, or even shorter than $\xi$, giving rise to metallic conductivity. Hence in this approach the MIC is mainly due to correlation and AF order, rather than disorder effect.

In this Report we generalize this approach to frequency-dependent phenomena and show the ACconductivity exhibits a maximum as function of frequency in an exact analogy with the DC conductivity maximum due to MIC. This outcome is fully understandable: In the presence of an external electromagnetic field of frequency $\Omega$, that frequency will replace the temperature $T$, playing the role of cutoff parameter, for $\Omega>T$ at low temperatures. Moreover, if we assume the AF correlation length $\xi$ is anisotropic, we argue that as a consequence the same is true for the MIC scale, then the in-plane conductivity anisotropy as well as the close parallel between the above two sets of experimental data can be interpreted in a self-consistent way.

Let us now analyze the theory in more details. In a gauge approach the physical resistivity is calculated using the Ioffe-Larkin addition rule: ${ }^{13} \rho=\rho_{s}+\rho_{h}$, where $\rho_{s}$ and $\rho_{h}$ are the spinon and holon resistivities, respectively. In order to evaluate the relevant current-current correlation functions, one first integrates over holons and spinons, and finds that the gauge propagator in the scaling limit, $\omega, q, \omega / q \rightarrow 0$, has a Reizer singularity 14 for the transverse component $\left\langle A^{T} A^{T}\right\rangle(\omega, \vec{q}) \sim\left(-\chi|\vec{q}|^{2}+i \kappa \omega /|\vec{q}|\right)^{-1}$ where $\kappa \sim \delta$ is the Landau damping.

The effect of Reizer singularity on gapless fermions is subdominant; it has been analyzed in Ref. 15 and at finite $T$ it gives a scattering rate for the holon of order $T^{4 / 3}$ instead of the usual Fermi liquid result $\sim T^{2}$. To include, non-perturbatively, the effect of gauge fluctuations in the spinon current-current correlation functions, we expand the spinon propagator in Feynman paths, as justified by the mass gap and we integrate over velocity fields in the eikonal approximation $\underline{12}$ Being gauge invariant, the correlator of the spinon current depends only on the gauge field strength. In the scaling limit only the magnetic components $F_{i j}$ are relevant (see Ref. 12); the corresponding propagator at finite $T$ is given by

$$
\begin{aligned}
& \left\langle F_{i j}(x) F_{r s}(0)\right\rangle=\left[\delta_{i r} \delta_{j s}-\delta_{i s} \delta_{j r}\right] \int \frac{d \omega}{2 \pi} \\
& \cdot \int \frac{d \vec{k}}{(2 \pi)^{2}} \frac{|\vec{k}|^{2} e^{i \vec{k} \cdot \vec{x}-i \omega x^{0}}}{i \frac{\omega}{|\vec{k}|} \kappa-\chi|\vec{k}|^{2}} \operatorname{coth}\left(\frac{\omega}{2 T}\right),
\end{aligned}
$$

where $x=\left(\vec{x}, x^{0}\right)$ with $x^{0}$ as the time variable. In the presence of an external electric field (the probe in linear response theory) with frequency $\Omega$, the integration over $\omega$ should be cutoff at $|\omega| \leqq \Lambda=\max (T, \Omega)$. (We further assume, for technical reasons, that $\Lambda x^{0} \ll 1$, as justified a posteriori, see Ref. 12).

Let us first focus on the static response and set $\Lambda=T$. As $\omega<T$, we approximate $\operatorname{coth} \frac{\omega}{2 T} \simeq \frac{2 T}{\omega}$, then the $\omega$ integration in (11) becomes

$$
\int_{0}^{T} \frac{d \omega}{2 \pi} \frac{2 T}{(\omega)^{2}+\left(\frac{\chi|\vec{k}|^{3}}{\kappa}\right)^{2}},
$$

eventually leading to an evaluation of the large-scale "magnetic" propagator: $-i(T / 4 \pi \chi) Q_{T}^{2} \exp \left(-Q_{T}^{2}|\vec{x}|^{2} / 4\right)$, where $Q_{T}=(T \kappa / \chi)^{1 / 3}$ is the inverse of an anomalous skin depth, identified as the length-scale of gauge fluctuations. The main effect of the gauge interaction on the spinon propagator is a renormalization of the mass term in the exponent in the limit $x_{0} \gg|\vec{x}|$ :

$$
m_{s} x^{0} \rightarrow \sqrt{m_{s}^{2}-\frac{T}{\chi} f\left(\frac{|\vec{x}| Q_{T}}{2}\right)} x_{0}-\frac{T}{2 \chi} Q_{T}^{2} g\left(\frac{|\vec{x}| Q_{T}}{2}\right) \frac{x_{0}^{2}}{m_{s}^{2}},
$$

where $f$ and $g$ are regular functions, whose explicit integral representations are given in Ref. 12. In the evaluation of the spatial Fourier transform of the currentcurrent correlator, the shift (3) eventually leads to a complex saddle point for $|\vec{x}| Q_{T}$ with absolute value $C \sim O(1)$ and phase factor $e^{i \pi / 4}$, which in turn introduces a dissipation term in the spinon gap:

$$
m_{s} \rightarrow M_{T}=\left(m_{s}^{2}-i c T / \chi\right)^{1 / 2},
$$

where $i c=f\left(C e^{i \pi / 4}\right)$. The competition between the gap term $m_{s}^{2}$ and the dissipation $T / \chi$ leads to a MIC upon the decrease of temperature, yielding a broad peak in the DC conductivity for $T \sim \chi m_{s}^{2} \sim(t / \delta)|\delta \ln \delta| \sim t|\ln \delta|$, thus shifting to lower temperature upon doping increase. More precisely the behavior derived for the DC conductivity is given by 12

$$
\sigma(T) \sim\left(\frac{\delta}{f^{\prime \prime}\left(C e^{i \pi / 4}\right)\left|M_{T}\right|}\right)^{1 / 2} \sin \left(\frac{1}{2} \arg M_{T}\right),
$$

where $f^{\prime \prime}$ means the second derivative.

We turn now to the AC conductivity at $T=0$ and set $\Lambda=\Omega$. In this case $\operatorname{coth} \frac{\omega}{2 T}$ is replaced by $\operatorname{sgn} \omega$ and the $\omega$-integration in (11) becomes:

$$
\int_{0}^{\Omega} \frac{d \omega}{2 \pi} \frac{\omega}{(\omega)^{2}+\left(\frac{\chi|\vec{k}|^{3}}{\kappa}\right)^{2}} .
$$

Up to logarithmic accuracy, one finds for the magnetic propagator at large scales: $-i(T / 4 \pi \chi) Q_{\Omega}^{2} \lambda \exp \left(-Q_{\Omega}^{2}|\vec{x}|^{2} / 4\right)$ where $Q_{\Omega}=(\Omega \kappa / \chi)^{1 / 3}$ and $0<\lambda \lesssim 1 / 2$, as follows from comparing (6) and (2). 
Repeating the steps of the DC calculations with this parameter $\lambda$ included, we find as the analog of (4):

$$
m_{s} \rightarrow M_{\Omega}=\left(m_{s}^{2}-i c \lambda \Omega / \chi\right)^{1 / 2} .
$$

For $\Omega \ll 2 m_{s}$, one easily obtains for the AC conductivity:

$$
\sigma_{1}(\Omega) \sim\left(\frac{\delta}{\lambda f^{\prime \prime}\left(C e^{i \pi / 4}\right)\left|M_{\Omega}\right|}\right)^{1 / 2} \sin \left(\frac{1}{2} \arg M_{\Omega}\right) .
$$

We see that the behavior of the $\mathrm{AC}$ conductivity at $T=0$ is rather similar to that of the DC conductivity, with a broad peak corresponding to a MIC, hardening and shifting to lower temperature upon doping increase, (see Fig. (1). Although the replacement of $T$ by $\Omega$ as cutoff yields a priori only an order estimation, the presence of the factor $\lambda$ (mainly coming from the factor $\operatorname{coth} \frac{\omega}{2 T}$ ) suggests that the position of the peak in the AC conductivity is shifted by a factor $\approx \lambda^{-1}$ to higher frequencies with respect to the DC case and its value is enhanced by a factor $\approx \lambda^{-\frac{1}{2}}$ ( see Fig. 22), roughly in agreement with experimental data $?^{7}$

The finite temperature behavior of the dynamical conductivity is as follows: for $T \ll \Omega$ we get only a small correction to the damping term in ( $\mathbf{7}$ ) of order $(T / \Omega)^{5 / 3}$ while for $\Omega \lesssim T$ essentially the $\Omega=0$ result applies and the conductivity will be frequency independent and equal to the DC value. Therefore upon temperature increase, the MIC peak is expected to shrink asymmetrically and eventually disappears from the spectrum, a behaviour consistent with experiments ${ }^{7}$. The limits of validity of the approximations involved in the calculation of the spinon correlation functions are $m_{s}^{2} \geq c \frac{\Lambda}{\chi} \geq m_{s} Q_{\Lambda}$, where the lower bound comes from the effectiveness of the saddle point at $|\vec{x}| \sim Q_{\Lambda}^{-1}$. When expressed in terms of temperature, this yields a range between a few tens and few hundreds of Kelvin. We expect that the upper limit corresponds to a crossover to a new "strange metal" phase, analyzed in $\frac{16}{6}$, where the $\pi$-flux lattice is melt and the "metallic", linear in $T$ resistivity is recovered. In some sense the pseudogap phase is on the "insulating" side of the MIC, and the description adopted here is a rather good approximation near the MIC, but the "high temperature asymptotics" $\sim T^{1 / 4}$ is not correct, although it reproduces at lower temperature the inflection point in resistivity found experimentally. The above calculations do not take into account the holon contribution to the physical conductivity, but that is of order $\Lambda^{4 / 3}$, hence negligible for small cutoff $\Lambda$.

Let us finally discuss how the in-plane resistivity anisotropy found 2.7 in untwinned single crystals of $\mathrm{La}_{2-x} \mathrm{Sr}_{x} \mathrm{CuO}_{4}(x=0.02-0.04)$ can fit into our scheme. The neutron scattering experiments have revealed incommensurate magnetic structure in lightly doped LSCO samples $(\delta \leq 0.05), \frac{17}{17}$ Unlike the superconducting LSCO compounds where the deviation of the elastic magnetic scattering peaks from $(\pi, \pi)$ is along the $a, b$ directions in the tetragonal basis,$\frac{18}{r}$ these peaks are rotated by
45 degrees around $(\pi, \pi)$, i.e., they are located along the $b^{*}$ axis in the orthorhombic basis. Moreover, from the half-width of the scattering peaks one can determine the magnetic correlation length in different directions. As a big surprise, one finds the correlation length strongly anisotropic. In particular, for $\delta=0.024, \xi_{a^{*}}^{\prime}=$ $94.9 A, \xi_{b^{*}}^{\prime}=39.9 A \stackrel{6}{ }$ The authors of Ref. 6 interpreted this result as due to stripe formation along $a$-axis, but no quantitative argument was given. This behavior is fully consistent with the magnetic susceptibility anisotropy, observed in untwinned lightly doped LSCO crystals (up tp $3 \%$ doping), 19 We do not have a quantitative microscopic theory to consider the anisotropy of the AF correlation length $\xi$, but we can see how such anisotropy can be included in our scheme and explore its consequences. Suppose the hole distribution is anisotropic (which may come from the underlying stripe structure), say the average distance between holes is bigger along the $a$ axis, so does the distribution of vortices on the AF background. To use the nonlinear- $\sigma$ model treatment of spinons, we can rescale the spatial coordinates. The result will be almost the same as in the isotropic case ${ }^{12}$ except for a coefficient $\alpha$ in the spinon dispersion $\sqrt{m_{s}^{2}+\alpha^{2} v_{s}^{2} k_{x}^{2}+v_{s}^{2} k_{y}^{2}}$, which reflects the ratio of the AF correlation lengths in different directions. Since the spinon mass $m_{s}$ is inversely proportional to the correlation length, we can effectively interpret this as $m_{s, a}<m_{s, b}$. To calculate the anisotropic conductivity we need to modify the entire scheme. However, the major effect can be grasped without detailed calculation. The diamagnetic susceptibility $\chi$ and Landau damping $\kappa$ due to holons will change, but only very slightly, since they come from angular integration. On the other hand, in the saddle point calculation of the path integral the effect is more pronounced, so the values of integrals $f, g$ and hence the numerical factor $c$, mentioned above, will also change. In our spin-charge gauge approach the combination $c \chi m_{s}^{2}=\frac{t}{6 \pi}|\ln \delta| r$ is crucial in determining the MIC scale. If we assume that the basic results of our theory developed for the $2 \mathrm{D}$ isotropic model survive the generalization to anisotropic case as outlined above, one would anticipate the parameter $r$ to be also anisotropic. In view of the AF correlation length anisotropy we expect $r_{a} / r_{b}<1$. As a consequence, the peak in $\sigma_{a}$ will be shifted to lower temperature with respect to $\sigma_{b}$, as follows from Eq. (4); the anisotropy ratio $\sigma_{a} / \sigma_{b}$ will show a sharp increase near the MIC and saturates as $T \rightarrow 0$, in agreement with experiments $\stackrel{2}{\underline{2}}$ The same phenomenon occurs for the $\mathrm{AC}$ conductivity at low temperatures, where the factor $\lambda$ makes the anisotropy ratio even bigger. To estimate this enhancement, we extract the ratio $r_{a} / r_{b}$ by fitting the DC data (the extracted value $\left.r_{a} / r_{b}=0.725\right)$. We can then use (8) to evaluate the corresponding anisotropy ratio for AC conductivity without introducing any additional parameters; a comparison with the experimental curve is shown in Fig. 3 This anisotropy is less pronounced than the experimentally observed value for AF correlation length, as quoted above for $\delta=0.024$, but this is consistent with the above 
scheme, where we expect that part of the anisotropy effect in the combination $c \chi m_{s}^{2}$ has already been cancelled by other effects.

It is true that these peculiar features in the in-plane conductivity are "due to modifications of the dynamics of the metallic carriers, and not due to the opening of a charge gap",$\frac{7}{2}$ since there is no charge gap in doped Mott insulators. What we have shown here is that these modifications are due to the presence of a gap in the spin excitations and its competition with the dissipation which is different from the disorder induced localization. For the same reason, the statement of only one mobility edge in the scaling theory $\stackrel{10}{=}$ does not apply here. As we learned from the authors of Ref. 7, 20 the anomalous behavior of $\mathrm{AC}$ conductivity is observed only up to $6 \%$ doping. It is understandable that the anisotropy due to stripes is not present since they are rotated by 45 degrees beyond $6 \%$ doping, and their orientation is alternating between $a$ and $b$ directions in adjacent layers. However, the disappearance of the low-frequency peak cannot be explained by the stripe interpretation. On the contrary, this is very natural in our interpretation since the MIC is not observed in samples beyond $6 \%$ doping in the absence of magnetic field. Now we make an explicit proposal: To do the $\mathrm{AC}$ experiment in the presence of a magnetic field which would suppress superconductivity and reveal MIC. If the deviation from the Drude behavior reappears, that would be a confirmation of our interpretation.

To conclude we have shown that the peculiar in-plane anisotropy of DC and AC conductivity observed in the lightly doped cuprates can be explained in a unified, self-consistent manner within the spin-charge gauge approach, and the key ingredient is to attribute the MIC to the correlation effect. The anisotropy of the AF correlation length, and consequently the MIC scale provides a rather natural explanation of the observed conductivity anisotropy, being most pronounced in the limit $\Omega, T \rightarrow 0$ which is very difficult to explain based only on the stripe existence. In fact, this is a crucial experiment to distinguish the disorder- and correlation-induced MIC.

We would like to sincerely thank Y. Ando and D. Basov for sending us the paper (Ref. 7) prior to publication and very helpful correspondence.
1 See, e.g., the review article: T. Timusk and B. Statt, Rep. Progr. Phys. 62, 61 (1999).

${ }^{2}$ Y. Ando, K. Segawa, S. Komiya and A. N. Lavrov, Phys. Rev. Lett. 88, 137005 (2002).

3 J. Zaanen and O. Gunnarson, Phys. Rev. B 40, 7391 (1989); H.J. Schulz, J. Phys. (Paris) 50,2833 (1989); D. Poilblanc and T..M. Rice, Phys. Rev. B 39, 9749 (1989); M.Cato and K. Machida, J. Phys. Soc. Jpn 59, 1047 (1990); S.A. Kivelson and V.J. Emery, in Strongly Correlated Electronic Materials: The Los Alamos Symposium 1993, (eds K. Bedell et al.)p. 619 (Addison-Wesley, Maine, 1994).

${ }^{4}$ S.R. White and D.J. Scdalapino, Phys. Rev. Lett. 81, 3227 (1998); A.L. Chernyshev, A.H. Castro Neto, and A.R. Bishop, Phys. Rev. Lett. 84, 4822 (2000).

${ }^{5}$ V.J. Emery, S.A. Kivelson, and O. Zachar, Phys. Rev. B 56, 6120 (1997); S.A. Kivelson, E. Fradkin, and V. Emery, Nature (London) 393, 550 (1998).

6 M. Matsuda et al., Phys. Rev. B 62,9148 (2000).

7 M. Dumm, D. N. Basov, S. Komiya, and Y. Ando, Phys. Rev. Lett. 91, 077004 (2003).

8 J. Zaanen, Science 286, 251 (1999).

9 See, e.g., D.N. Basov et al. Phys. Rev. B 49, 12165 (1994); ibid, Phys. Rev. Lett. 81, 2132 (1998).

${ }^{10}$ W. Apel and T.M. Rice, J. Phys. C: Solid State Phys. 16, L1151 (1983); I. Zambetaki et al., Phys. Rev. Lett. 76, 3614 (1996).

11 P.A. Marchetti, Z.B. Su, L. Yu, Phys. Rev. B 58, 5808 (1998).

12 P.A. Marchetti, J.H. Dai, Z.B. Su, and L. Yu, J. Phys. Condensed Matter 12, L329 (2000) ; P.A. Marchetti, Z.B. $\mathrm{Su}$ and L. Yu, Phys. Rev. Lett. 86 (2001) 3831 ; P.A. Marchetti, L. De Leo, G.Orso, Z.B. Su, L. Yu, Phys. Rev. B. 69, 024527 (2004).

13 L. Ioffe and A. Larkin, Phys. Rev. B 39,8988 (1989).

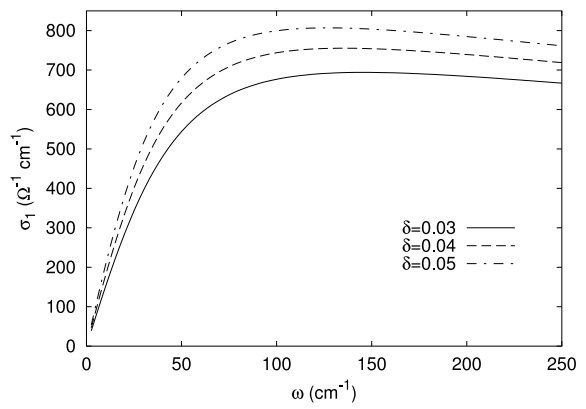

FIG. 1: Theoretically calculated frequency dependence of the AC conductivity for different dopings: $\delta=0.03$ (full line), $\delta=0.04$ (dashed) and $\delta=0.05$ (dotted).

14 M. Reizer, Phys. Rev. B 40, 11571 (1989).

15 P.A. Lee and N. Nagaosa, Phys. Rev. Lett. 65, 2450 (1990); Phys. Rev. B 46, 5621 (1992); L.B. Ioffe and P.B. Wiegmann, Phys. Rev. Lett. 65, 653 (1990).

16 P.A. Marchetti, G.Orso, Z.B. Su, L. Yu, in preparation.

17 S. Wakimoto et al. Phys. Rev. B 60, R769 (1999); ibid, Phys. Rev. B 61, 3699 (2000).

18 K. Yamada et al., Phys. Rev. B 57, 6165 (1998).

19 A.N. Lavrov, Y. Ando, S. Komiya, and I. Tsukada,Phys. Rev. Lett. 87, 017007 (2001).

20 D.N. Basov, private communication. 


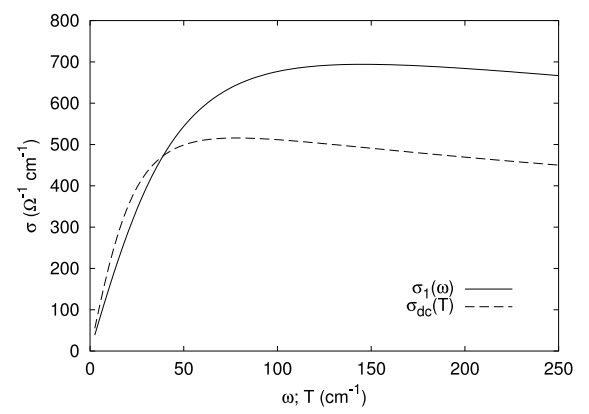

FIG. 2: Calculated frequency dependence of the AC conductivity for $\delta=0.03$. Also shown is the corresponding DC conductivity as a function of temperature (in $\mathrm{cm}^{-1}$ ).

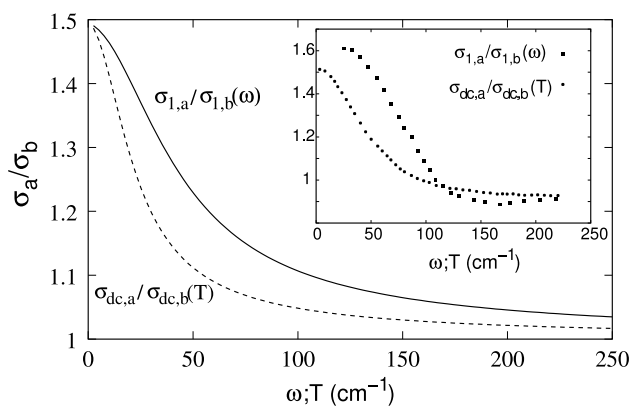

FIG. 3: Calculated frequency dependence of the AC conductivity anisotropy ratio for $\delta=0.03$. The corresponding DC ratio as a function of temperature $\left(\right.$ in $\mathrm{cm}^{-1}$ ) is shown with dashed line. Inset shows the corresponding DC and AC data, taken from Refs 2 and 7 , respectively. 